\title{
End-Stage Renal Disease and Early-Onset Calciphy- laxis: A Case Report
}

\begin{abstract}
Keywords: Calciphylaxis; Sodium thiosulfate; End-stage renal disease

Abstract

We discuss the presentation of $\mathrm{Mr}$. B, a 51-year-old African American male, diagnosed with End-Stage Renal Disease (ESRD) initiated on hemodialysis 6 months prior to presentation. After starting dialysis, the patient developed a bulla measuring approximately $8 \mathrm{~cm}$ $\times 10 \mathrm{~cm} \times 2 \mathrm{~cm}$, which rapidly progressed into necrotic ulcerations of the lower extremities. Work-up of the ulcerations were consistent with severe calciphylaxis. He developed these lesions just 6 months into his end-stage renal disease diagnosis. Unique to this case is the rapidity of onset, severity and extent of lesions and positive response to sodium thiosulfate. Calciphylaxis as an entity is not well understood, and there is limited evidence behind current treatments.
\end{abstract}

\section{Objective}

This case highlights a dramatic example of calciphylaxis. The presentation of this patient is especially important due to the rapid progression of tissue damage. In presenting this case, we aim to bring attention to this debilitating entity, and to emphasize the need for early diagnosis to implement treatment in the hope of positively impacting prognosis and quality of life.

\section{Clinical Case}

$\mathrm{MB}$ is a 51 year old African American male with a past medical history significant for end-stage renal disease, aortic aneurysm, chronic gout, hypertension, obesity with a BMI of $33.5(108.5 \mathrm{~kg})$, anemia of chronic kidney disease, hyperlipidemia, and methicillin resistant Staphylococcus aureus colonization. In June 2016, Mr. B began dialysis for end-stage renal disease that he received every Tuesday, Thursday and Saturday. His medications included non-calcium containing phosphate binders $800 \mathrm{mg}$ PO TID and darbepoetin alfa $60 \mathrm{mcg}$ (0.3 mL IV Q7 days) for hemodialysis, carvedilol $25 \mathrm{mg}$ PO BID and hydralazine $50 \mathrm{mg}$ PO TID for hypertension. On July 29, 2016, a short time after starting hemodialysis, Mr. B developed a bulla on his right lower extremity measuring approximately $8 \mathrm{~cm} \times 10 \mathrm{~cm}$ x $2 \mathrm{~cm}$. Over a 24 hour period, he developed bullae that started to drain leading to a concern for an abscess. He was soon scheduled for surgical debridement and the tissue samples and culture of the draining wound were sent to pathology revealing extensive purulent necrotic tissue with surrounding calcium deposition consistent with calciphylaxis. The wounds were unusually extensive, measuring 10 $\mathrm{cm} \times 7 \mathrm{~cm} \times 1.5 \mathrm{~cm}$ on the right posterior thigh, $12 \mathrm{~cm} \times 5 \mathrm{~cm} \times 1.5$ $\mathrm{cm}$ on right calf, $6 \mathrm{~cm} \times 5 \mathrm{~cm} \times 1 \mathrm{~cm}$ on the left lower extremity. His wound cultures grew methicillin resistant Staphylococcus aureus (Figure 1).

Throughout Mr. B's treatment, he was followed by a nephrologist who managed his ESRD as well as his calciphylaxis. As part of his treatment for calciphylaxis, he was receiving sodium thiosulfate $25 \%$

\section{Journal of}

Clinical \& Medical Case Reports

Brielle A. Marks*, Janel M. Bakou and Ahmed Ibrahim The Department of Internal Medicine, Northwest Hospital, USA Address for Correspondence:

Brielle A. Marks, Department of Internal Medicine, Northwest Hospital, 5401 Old Court Road, Randallstown, USA, E-mail: briellealanamarks@gmail.com

Submission: 20 December, 2016

Accepted: 02 February, 2017

Published: 08 February, 2017

Copyright: ( 2017 Marks BA, et al. This is an open access article distributed under the Creative Commons Attribution License, which permits unrestricted use, distribution, and reproduction in any medium, provided the original work is properly cited.

IV solution and Gentamicin $160 \mathrm{mcg}$ ( $4 \mathrm{~mL}$ IV) Tuesday, Thursday, Saturday, and Collagenase Q day. About three months into his treatment, Mr. B presented to our hospital from his extended care nursing facility for evaluation of lower extremity pain preventing compliance with hemodialysis. The patient required a Hoyer lift for transfers and transport to hemodialysis, but the pain had resulted in his inability to receive hemodialysis for four days. Consequently, he was sent to our hospital on October 1, 2016. On physical exam, the patient had extensive wound wrappings covering both of his lower extremities and a portion of his lower abdomen. He was, therefore, admitted to the Internal Medicine floor for continuation of hemodialysis and management of his wounds due to calciphylaxis.

During hospitalization various consultants co-managed the case with the admitting service. Nephrology, general surgery, and infectious disease played an important role in care management. Nephrology recommended he continue with hemodialysis and receive sodium thiosulfate $25 \%$ IV every Tuesday, Thursday and Saturday as recommended by his previous nephrologist as well as non-calcium containing phosphate binders as treatment for his calciphylaxis. Surgery recommended wound debridements, but the patient refused and local wound care was done by nursing. Pain management was also warranted providing $2 \mathrm{mg}$ of hydromorphone, as needed, as well as a bolus dose before wound care. Blood work was taken daily to

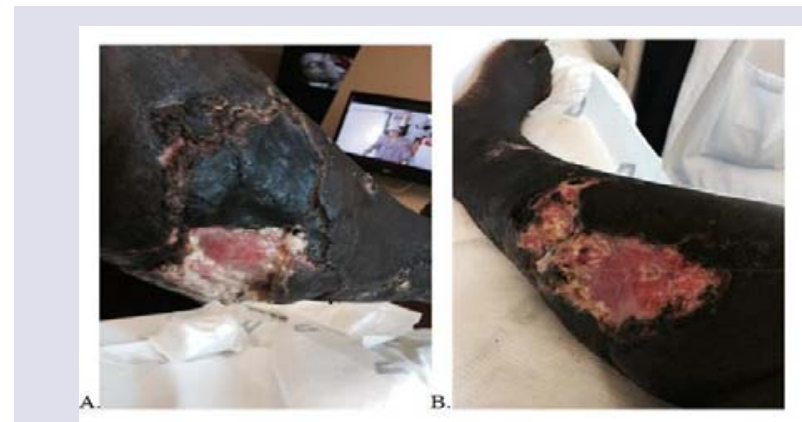

Figure 1: A) Initial stage of necrotic poor healing wound of the left calf before surgical debridement taken on August 3, 2016. B) Initial stage of necrotic poor healing wound of the right calf after first surgical debridement taken on August 3, 2016. 

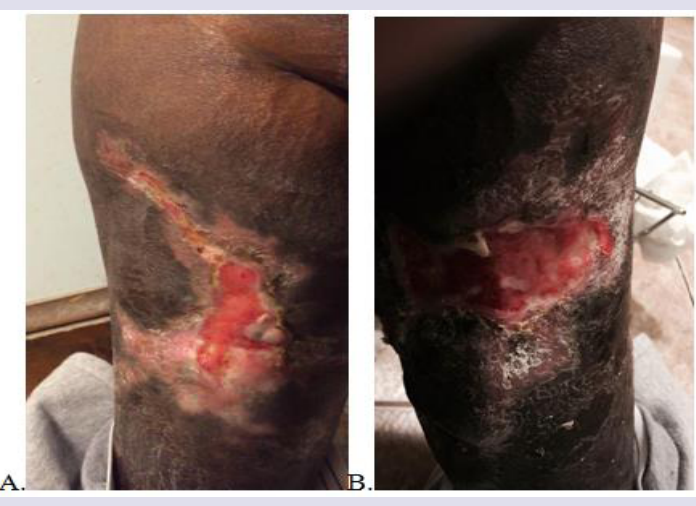

Figure 2: Healing wounds on the lower extremities of the patient. The patien is being treated with STS and collagenase for wound care.

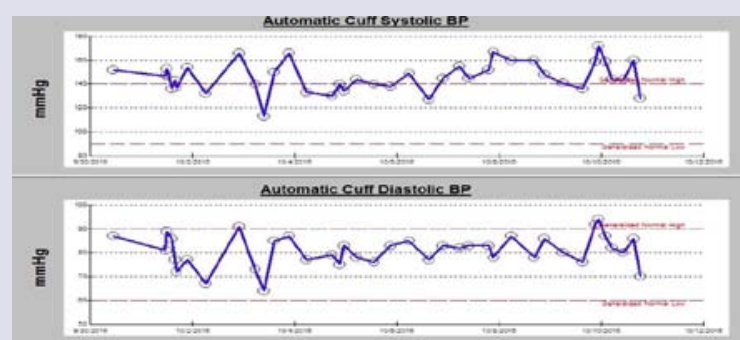

Graph 1: Graph shows a trend of systolic and diastolic blood pressure throughout the patient's stay at the hospital.

monitor his electrolytes, along with a daily $\mathrm{CBC}$. Heparin was given from the time of admission per DVT prophylaxis protocol. 5,000 units of Heparin were given subcutaneously three times a day. The patient's comorbidities were also monitored and managed during his inpatient stay. His blood pressure was taken daily and is plotted on the graph below (Graph 1):

Throughout Mr. B's treatment process, his phosphate levels were measured serially in order to monitor his progress (Graph 2). Phosphate levels are important to follow because high levels of serum phosphate lead to an increase in serum calcium from bone, and therefore, cause calcium deposition into the arterioles. As demonstrated by Graph 2, the majority of Mr. B's phosphate levels are well over the normal range (2.4-4.3 mEq is the range used).

Along with phosphate levels, Parathyroid Hormone (PTH) and calcium levels were measured three times throughout his treatment process (Graph 3). His serum PTH values were $120 \mathrm{mEq}$ in July, 107 $\mathrm{mEq}$ in August, and $40 \mathrm{mEq}$ in October, representing two elevated PTH levels and one normal. Calcium levels measured $9.5 \mathrm{mEq}$ in July, 9.0 in August, and 10.5 in October.

Mr. B continued to receive treatment from nephrology and the wound care team. In addition, a blood culture and swab of the wounds were taken due to increasing leukocytosis. On October 4, 2016, infectious disease was consulted for positive wound cultures, and bacteremia with Acinetobactor baumannii. Per their recommendation, he was started on vancomycin and piperacillintazobactam. Despite wound care and antibiotics, the calciphylaxis spread rapidly to his abdomen, back and sacrum.

Due to the relentless progression of his calciphylaxis-associated non-healing ulcers, the attending physician thought it would be best to discuss prognosis with the patient and his spouse. Mr. B was informed that his prognosis is poor and recommendations for surgical debridement were made based on the antibiotic resistant blood cultures. Mr. B understood the risks and the benefits of surgery versus empiric antibiotic treatment, but opted to only consider the debridement if it were in an emergent situation. At this point, the patient was felt to have maximized the benefit of hospitalization and was transferred back to his extended care nursing facility for ongoing wound care needs and continued hemodialysis treatment.

To date, Mr. B's extensive wounds are healing. After discharge from the hospital and his infection was controlled, he underwent the surgical debridement that was recommended. His wounds were treated with silver gluconate, calcium gluconate, and a wet-to-dry method. He is now living at home and is remarkably able to ambulate. $\mathrm{He}$ is still receiving sodium thiosulfate as part of his wound care treatment and is followed by nephrology (Figure 2).

\section{Discussion}

The pathogenesis of calciphylaxis is relatively unknown, however, one of the commonly agreed upon theories is the deposition of calcium in the intima of the arterioles causing tissue ischemia and necrosis [1]. Elevated serum phosphate, common in End-Stage Renal Disease (ESRD), leads to an increase in serum calcium from bone, thereby causing deposition into the arterioles. According to Jeong et al. the transformation begins by the conversion of vascular smooth muscle cells to cells phenotypically similar to osteoblastic cells [2]. For reasons not fully elucidated, this transformation is initiated by environmental conditions under the presence of uremia, reactive oxygen species, and elevated phosphate levels. As a result, there is also a decrease in the matrix gla protein, which normally functions to inhibit calcium deposition [2]. This transformation leads to the buildup of calcium in the intima leading to ischemia and necrosis of the arteriole [3]. Calciphylaxis manifests predominantly in the distal extremities as well as other body parts with high fat content like the back, abdomen, and thighs. Initial presentation starts with a painful, violet colored skin lesion with vesicles appearing near the surrounding ulcer. Rapid progressions of these wounds are common with a presenting black eschar and necrotic tissue found on the affected area [3].

There is a multifactorial component of calciphylaxis with increased risk factors associated with, end-stage renal disease,

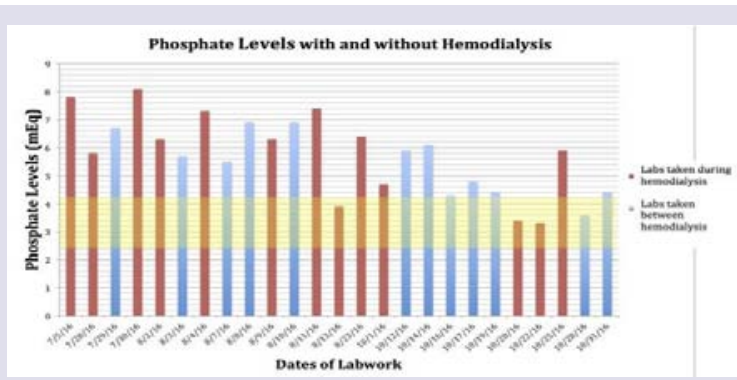

Graph 2: The graph above illustrates Mr. B's phosphate levels throughout his treatment, during and between hemodialysis. The yellow box in the middle of the graph represents the hospitals range for normal phosphate levels and ranges between 2.4-4.3 mEq. As demonstrated by the graph, the majority of the patient's lab values are well above the normal range. 


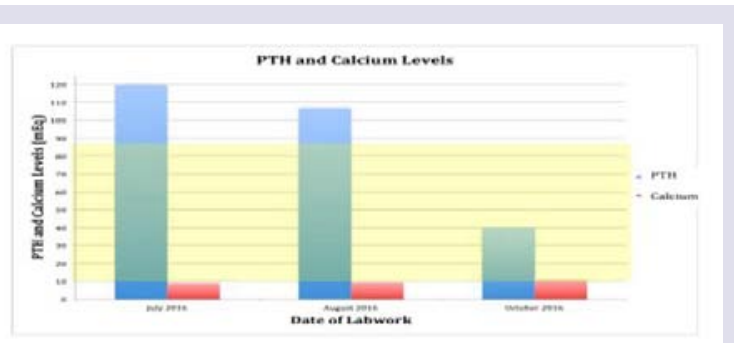

Graph 3: This graph demonstrates the patient's parathyroid hormone (PTH) and calcium levels. Normal range used for PTH and calcium were $12-88 \mathrm{mEq}$ and $8.5-10.1 \mathrm{mEq}$ respectively. The graph shows that two of three values for PTH were well above the upper limit of normal.

hypercoagulability, female gender, obesity, hyperparathyroidism, average age of 60 , hypertension, the use of Warfarin, underlying malignancy, and patients with increased inflammatory response [4]. Patients may experience hypercalcemia, hyperphosphatemia, and elevated parathyroid hormone, however, some patients may not have an elevation in any of these [3]. In a study conducted by McCarthy et al. 101 patients who were diagnosed with end-stage renal disease and exhibited any of the risk factors listed above were monitored over their disease process to determine the percentage of patients who developed calciphylaxis [5]. Patients with obesity were $68.0 \%$ more likely to develop calciphylaxis and patients with hypertension were $78.2 \%$ more likely to develop calciphylaxis [5]. Furthermore, patients who received Warfarin had $62.4 \%$ increased risk, and patients who received other anticoagulation treatments had a $19.8 \%$ chance for development. Our patient having both, hypertension and a BMI in the obese range had increased his risk for development of the disease. Along with these pre-existing factors, our patient also received Heparin as a form of anticoagulation during his treatment.

In addition to this list, vitamin-D and calcium-containing medications used for the sequestration of phosphate, can also put a patient at risk for calciphylaxis [4]. Hooman et al. found that treating their patients with calcium containing products greatly increased the severity of the disease [4]. With that being said, it is important to consider other pharmaceutical options with similar mechanisms of action without adding additional calcium to the patient's serum.

A diagnosis for calciphylaxis can be difficult and there is no specific laboratory test that confirms the diagnosis. A diagnosis is made clinically and confirmed with pathological tissue biopsy. A biopsy shows multiple depositions of calcification and fibrinous thrombi throughout the vessel tissue. A positive biopsy, as well as a lack of anti-inflammatory cells in the tissue, confirms the diagnosis [3]. Radiologic testing can aid in the diagnosis of calciphylaxis. Plain X-ray and a nuclear bone-scan can both be used; however, radiographic imaging is not recommended for diagnostic purposes. The value of other diagnostic modalities such as serum markers are mainly used in the academic setting, however they are currently not recommended as the primary diagnostic factor for calciphylaxis. Basic workup may include assessment of renal function, mineral bone chemistry, liver function, coagulation factors, and inflammatory cytokines [6]. These are important markers to take into consideration since most indicate a predisposition of potential risk factors.

Treatment for calciphylaxis requires the coverage of normalizing serum levels of calcium, phosphorus, and parathyroid hormone as well as pain management and wound care [1]. There are several successful treatment options for calciphylaxis such as Sodium Thiosulfate (STS), hyperbaric oxygen chamber, and bisphosphonates, but the most successful and approved for treatment and prophylaxis is Sodium Thiosulfate (STS) [5]. This treatment is typically the treatment of choice for patients who do not have hyperparathyroidism [1]. This medication is believed to work by dissolving the deposited calcium in the tissue resulting in a more soluble form-calcium thiosulfate. This end product then works as antioxidant in the tissues and has vasodilatory effects. In McCarthy et al. a study showed that patients who received STS during their treatment did not show a significant impact on overall mortality. The study showed that there was a 3 year survival rate of $32.8 \%$ for patients who did receive the treatment versus a $28.0 \%$ survival of those who did not $(\mathrm{HR}=1.22$; 95\% CI; 0.67 2.22; $\mathrm{P}=0.51)[5]$.

Long term response of STS is determined by the pain relief after use during the first two weeks of treatment. Doses of the treatment are recommended to be adjusted depending on the amount of dialysis the patient receives throughout the week. STS $25 \mathrm{~g}$ is typically given within the last half hour of dialysis with a $100 \mathrm{ml}$ normal saline drip. STS should be given with every hemodialysis session. This is recommended dose for patients who are $70 \mathrm{~kg}$ or greater and receiving hemodialysis three times per week. Dose adjustments should be made for patients who are receiving hemodialysis more frequently, patients who weigh $60 \mathrm{~kg}$ or less should receive $12.5 \mathrm{~g}$ instead of $25 \mathrm{~g}$, or if a patient is on renal replacement therapy, in order prevent increased risk for adverse side effects [6].

Hyperbaric oxygen chamber applies $100 \%$ oxygen in an airtight therapy bed which transmits increased oxygenation to the affected tissues. Sessions in the hyperbaric oxygen chambers typically last 90 minutes. The patient is recommended to receive 40 to 60 treatments under 2 to 2.5 atmospheres [7,8]. In a study using 46 patients who received hyperbaric oxygen chamber treatment for calciphylaxis, Jeong et al. determined that $58 \%$ of patients showed improvement in their wounds. Of this $58 \%$ of the patients, about half of the patient's wounds resolved completely [2].

Bisphosphonates promote the function of osteoblasts while inhibiting osteoclast function. This treatment has been successful in calciphylaxis patients, especially in the adult population with endstage renal disease [4]. The use of bisphosphonates is typically used to prevent hypercalcemia and is especially useful in diseases that have an increase in calcium and phosphate [2]. Another way bisphosphonates have been successful in controlling calcium and phosphate is by inhibiting the effects of Parathyroid Hormone (PTH). Specifically in patients with calciphylaxis, bisphosphonates can be used to control the levels of calcium and phosphate by controlling the PTH [2].

Bisphosphonates are also believed to aid in the healing process of calciphylaxis. Although not completely understood, it is believed that through the alteration of the calcium hydroxyapatite formation, adhering to the vascular smooth muscle cells that are phenotypically similar to osteoblasts and osteoclasts, in addition to several other pathways [2].

Whether to consider surgical debridement, is a complicated decision and is left up to the surgeon. This modality may be risky given that it predisposes the patient to wound infections and poor wound healing. The treatment plan should include wound care and the treatment team should strive prevent infection, promote proper wound healing, and keep the wound clear of necrotic tissue 
[5,6]. In a retrospective analysis conducted by the Mayo Clinic, surgical wound debridement showed a $61.1 \%$ survival rate after one year compared to $27.4 \%$ who did not receive surgical debridement $(\mathrm{P}=0.008)[6,9]$. Nigewaker et al. suggests wound debridement in conjunction with negative pressure therapy in patients with infected tissue. This approach promotes a healthy wound bed that can receive debridement and can receive skin graft [6]. If the patient does not have infected wounds, the larger necrotic wounds may have poor healing properties and an increased risk for infection [6].

Maggot debridement is an alternative to surgical debridement. The maggots carefully debride the necrotic tissue, while also preventing infection and encouraging proper wound healing [2]. As per Jeong et al. maggot therapy is time consuming, pain inducing and limited research has been done on this approach in calciphylaxis wound care [2]. Patients who are receiving anticoagulation therapy are at greater risk for bleeding during the use of maggot debridement.

Calciphylaxis warrants a poor prognosis. According to Weenig et al. the average life expectancy after the diagnosis of calciphylaxis is 2.6 months to 10.8 months [9]. The most common cause of death in cases of calciphylaxis is sepsis due to its link to gangrenous necrosis [1]. Weenig et al. determined that in a study of 64 patients with calciphylaxis that causes other than sepsis included progression of the calciphylaxis, renal failure, myocardial infarction, and multipleorgan failure [9]. The length of time between a patient receiving dialysis and the subsequent development of calciphylaxis has been on average, 3.1 years. Longer time spans have been reported, up to 7 years, however, there are case reports of shorter time spans to the onset of calciphylaxis after dialysis [6].

Our patient represents a case unique in that his calciphylaxis presented unusually early into his diagnosis of end-stage renal disease, the rate of progression of his disease and the extensiveness of his calciphylaxis. He also had a remarkable response to STS. For this reason, more studies should be done to analyze treatment efficacy and the consideration of calciphylaxis as a diagnosis early to improve prognosis.

\section{Conclusion}

The pathophysiology of calciphylaxis is not completely understood, but there are multiple known risk factors that should prompt treating physicians to consider it. Calciphylaxis should be considered in cases of wound necrosis refractory to treatment. Our patient had predisposing risk factors such as obesity and end-stage renal disease that put calciphylaxis high in consideration amongst the potential etiologies of his wounds. He started receiving STS early in his treatment as recommended by his nephrologist. Despite his presenting condition in October to our hospital, to date our patient is currently healing and doing well. While on dialysis in the hospital, the patient's phosphate continued to carry a high trend while even on non-calcium containing phosphate binders. One treatment option that may have been considered is the addition of a bisphosphonate to his treatment plan. Bisphosphonates would have potentially helped keep his phosphate levels under control as well as his PTH. In addition to our patient's treatment, he was not a candidate for hyperbaric oxygen therapy due to the extensiveness of his wounds, inability to ambulate, and his antibiotic resistant infection. Given that Mr. B did refused surgical debridement, maggot therapy might have been an alternative for him as well. Since maggot therapy helps to promote proper wound healing and decreases the risk of infection, it may have been safer to consider this type of debridement to prevent further spread of the antibiotic resistant infection Mr. B possessed.

An early diagnosis of calciphylaxis allows for appropriate discussions surrounding prognosis, and the ability to take measures that may impact a patient's quality of life. Mr. B presented with physical findings of calciphylaxis and he was diagnosed early in his treatment regimen. He is now healing appropriately and improving in his care. STS was implemented immediately upon diagnosis and continued throughout his treatment process. We would recommend further studies be conducted on patients with calciphylaxis and different treatment options. This case also illustrated the need for further studies to determine effective treatment options.

\section{Informed Consent}

The patient has been made aware of this research paper and the use of personal medical information. All terms and agreements have been discussed with the patient and the patient has agreed to these terms.

\section{References}

1. Garcia CP, Roson E, Peon G, Abalde MT, De La Torre C (2013) Calciphylaxis Treated with Sodium thiosulfate: report of two cases. Dermatol Online J 19: 19616

2. Jeong HS, Dominguez AR (2016) Calciphylaxis: controversies in pathogenesis, diagnosis, and treatment. Am J Med Sci 351: 217-227.

3. Martin R (2004) Mysterious calciphylaxis: wounds with eschar--to debride or not to debride?" Ostomy Wound Manage 50: 64-70.

4. Hooman N, Naghshi-Zadiyan R, Mehrazma M, Jahangiri F (2015) Successful treatment of calciphylaxis with pamidronate. Iran J Kidney Dis 9: 67-70.

5. McCarthy JT, El-Azhary RA, Patzelt MT, Weaver AL, Albright RC, et al. (2016) Survival, risk factors, and effect of treatment in 101 patients with Calciphylaxis. Mayo Clin Proc 91: 1384-1394.

6. Nigwekar SU, Kroshinsky D, Nazarian RM, Goverman J, Malhotra R, et al. (2015) Calciphylaxis: risk factors, diagnosis, and treatment. Am J Kidney Dis 66: $133-146$.

7. Wangen T, Anderson S, Fencl K, Mangan S (2014) Calciphylaxis: an unusual case with an unusual outcome. Am J Nurs 114: 24-31.

8. Bliss DE (2002) Calciphylaxis: what nurses need to know. Nephrol Nurs $J$ 29: 433-438.

9. Weenig RH, Sewell LD, Davis MD, McCarthy JT, Pittelkow MR (2007) Calciphylaxis: natural history, risk factor analysis, and outcome. J Am Acad Dermatol 56: 569-579.

Acknowledgements

We thank Northwest Hospital for their support in this paper 